\title{
Estudo da vascularização arterial em corações de capivara (Hydrochaeris hydrochaeris - CARLETON, M. D. 1984)
}

\author{
Study of the arterial vascularization in capibara hearts (Hydrochaeris hydrochaeris - \\ CARLETON, M. D. 1984)
}

\author{
Suziellen Cristina TENANI ${ }^{1}$; Alan Peres Ferraz de MELO²$^{2}$; Rosângela Felipe RODRIGUES ${ }^{2}$ \\ ${ }^{1}$ Médica Veterinária Autônoma, São José do Rio Preto-SP \\ ${ }^{2}$ Curso de Medicina Veterinária do Centro Universitário de Rio Preto, São José do Rio Preto-SP
}

\begin{abstract}
Resumo
Utilizaram-se 30 corações de capivara (Hydrochoerus hydrochoeris), entre machos fêmeas de diferentes faixas etárias, cedidos pela Empresa Pró-Fauna, cidade de Iguape, estado de São Paulo, registrado no IBAMA sob n $235930848-0$ e no SIF sob $\mathrm{n}^{\circ} 3381$. As artérias coronárias direita e esquerda foram canuladas e injetadas com substância látex corada com pigmento específico. Em 96,9\%s dos corações estudados, encontrou-se as artérias coronária direita e esquerda que possuem origem a partir da aorta ascendente. Em 3,3\% dos casos não há artéria coronária esquerda, mas sim os ramos interventricular paraconal e circunflexo. Quanto ao ramo intermédio, este pode apresentar origem a partir do ramo interventricular paraconal em $40 \%$ dos casos, ou pode apresentar sua origem diretamente da aorta ascendente em $3,3 \%$ dos casos; e, ainda, pode ter origem a partir do ramo circunflexo em $56,6 \%$ dos casos. Quanto à artéria coronária direita, esta possui menor calibre que a artéria coronária esquerda em 100\% dos casos. Apresenta-se percorrendo o sulco coronário emitindo ramos para a margem ventricular direita. Na face atrial, a referida artéria coronária apresenta ramo anastomótico, em todos os casos, como descrito anteriormente.
\end{abstract}

Palavras-chave: Artéria coronária. Coração. Capivara.

\begin{abstract}
The present study used thirty randomly selected capybara (Hydrochaeris hydrochaeris) hearts, among males and females with different ages with no sex or age selection, gently donated by Empresa Pró-Fauna, city of Iguape, in São Paulo State. With IBAMA register number 235930848-0 and SIF register number 3381. Catheters were put into the right and left coronary arteries and a latex substance stained with a specific pigment was injected into them. We found in $96.9 \%$ of the hearts studied that the right and left coronary arteries had origin from the ascending aorta artery. In 3.3\% of the hearts studied the right coronary artery wasn't present, but only the paraconal interventricular and circumflex branches. The intermediary branch had originated from the paraconal interventricular branch in $40 \%$ of the heart samples, or could have its origin directly from the ascendant aortic artery in $3.3 \%$ of the hearts samples; and finally could have originated from the circumflex branch in $56.6 \%$ of the heart samples. The right coronary artery had smaller caliber than the left coronary artery in all the samples / 100\% of the heart samples. It went through the coronary channel with branches being emitted to the right ventricular edge. In the atrial face side this artery had anastomotic branch in all the samples, as previously described.
\end{abstract}

Keywords: Conary artery. Heart. Capybara.

\section{Introdução}

O coração de animais silvestres tem sido amplamente estudado: Borelli, Fernandes Filho e Ferreira ${ }^{1}$, estudando búfalo, Bezerra et al. ${ }^{2}$, estudando camelo, Vicentini, Orsi e Dias ${ }^{3}$, estudando cobaia.
Correspondência para:

Alan Peres Ferraz de Melo

Rua Yvette Gabriel Atique, 45, Bairro Boa Vista

São José do Rio Preto - SP, 15025-400

E-mail: alanmelo@unirp.edu.br

Recebido: 16/10/2008

Aprovado: 04/03/2010 
Visando este aspecto, tem-se por objetivo no presente trabalho estudar a arquitetura das artérias coronárias, tentando descrever sua distribuição na parede cardíaca, seu padrão fluxográfico coronariano, delimitar as diversas áreas vasculares e evidenciar ou não anastomoses entre seus diversos ramos.

\section{Material e Método}

Paraarealizaçãodopresentetrabalhoforamutilizados 30 corações de capivara (Hydrochoerus hydrochoeris), entre machos fêmeas de diferentes faixas etárias, cedidos pela Empresa Pró-Fauna, cidade de Iguape, estado de São Paulo, registrado no IBAMA sob $\mathrm{n}^{\circ}$ 235930848-0 e no SIF sob n 3381.

Após o abate dos animais, os conjuntos coração e pulmões foram separados do restante das demais vísceras. Nas próprias dependências do abatedouro o material foi lavado em água corrente com o objetivo de retirar o excesso de sangue. Em seguida, separouse o coração do pulmão. As artérias coronárias direita e esquerda foram canuladas e inicialmente realizada perfusão com solução fisiológica ligeiramente aquecida, com objetivo de retirar o máximo ainda presente de sangue destas artérias. Em seguida, injetou-se substância látex (substância látex extraída da Hevia brasiliensis, obtida na Usina de Beneficiamento de Látex Borrachas Bonilha, Mirassol-São Paulo) corada com pigmento específico (Suvinil Corante, Glasuriti do Brasil S.A.) nas artérias previamente canuladas, onde se obteve o preenchimento dos vasos do referido órgão. Logo após a injeção da substância látex, os corações foram preenchidos internamente com algodão para conservar seu formato. Então os corações já injetados foram transportados para o Laboratório de Anatomia Veterinária do Curso de Medicina Veterinária do Centro Universitário de Rio Preto, Município de São José do Rio Preto, Estado de São Paulo onde foram colocados em solução aquosa de formaldeído a $20 \%$, por período não inferior a 72 horas. Após este período, os corações foram dissecados nas suas faces auriculares e atrial.

Quanto à nomenclatura das estruturas encontradas, foi utilizada a Nomina Anatomica Veterinaria ${ }^{4,5,6}$.

\section{Resultados}

Em 96,9\%s dos corações estudados encontraram-se as artérias coronária direita e esquerda que possuem origem a partir da aorta ascendente (Figuras 1A, C e D). Em 3,3\% dos casos não se evidenciou a artéria coronária esquerda, mas sim o ramo interventricular paraconal e o ramo circunflexo. Nos casos onde a artéria coronária esquerda está presente, ela se divide em ramo interventricular paraconal e ramo circunflexo. O ramo interventricular paraconal apresentase percorrendo o sulco interventricular paraconal em direção ao ápice do coração (Figuras 1C e D). Este ramo emite durante o seu trajeto vários ramos que se distribuem no parênquima do órgão (Figuras $1 \mathrm{C}$ e D), sendo o ramo intermédio o maior, podendo apresentar origem a partir do ramo interventricular paraconal em $40 \%$ dos casos (Figura 1C), ou pode vir diretamente da aorta ascendente em 3,3\% dos casos, ou, ainda, a partir do ramo circunflexo em 56,6\% dos casos (Figura 1D). Uma vez possuindo origem a partir do ramo circunflexo, esta origem pode ser visível pela face auricular em 36,6\% (Figura 1D), ou pela margem caudal em $13,3 \%$, ou ainda pela face atrial em $6,6 \%$ dos casos.

O ramo circunflexo percorre o sulco coronário em direção à margem ventricular esquerda, indo ocupar o sulco interventricular subsinuoso, emitindo vários ramos arteriais para o átrio e ventrículo em $100 \%$ dos casos (Figura 1B). O ramo circunflexo, logo em seu início, apresenta um ramo anastomótico com a artéria coronária direita em 100\% dos casos (Figura 1B).

Quanto à artéria coronária direita, possui menor diâmetro que a artéria coronária esquerda em $100 \%$ dos casos. Apresenta-se percorrendo o sul- 


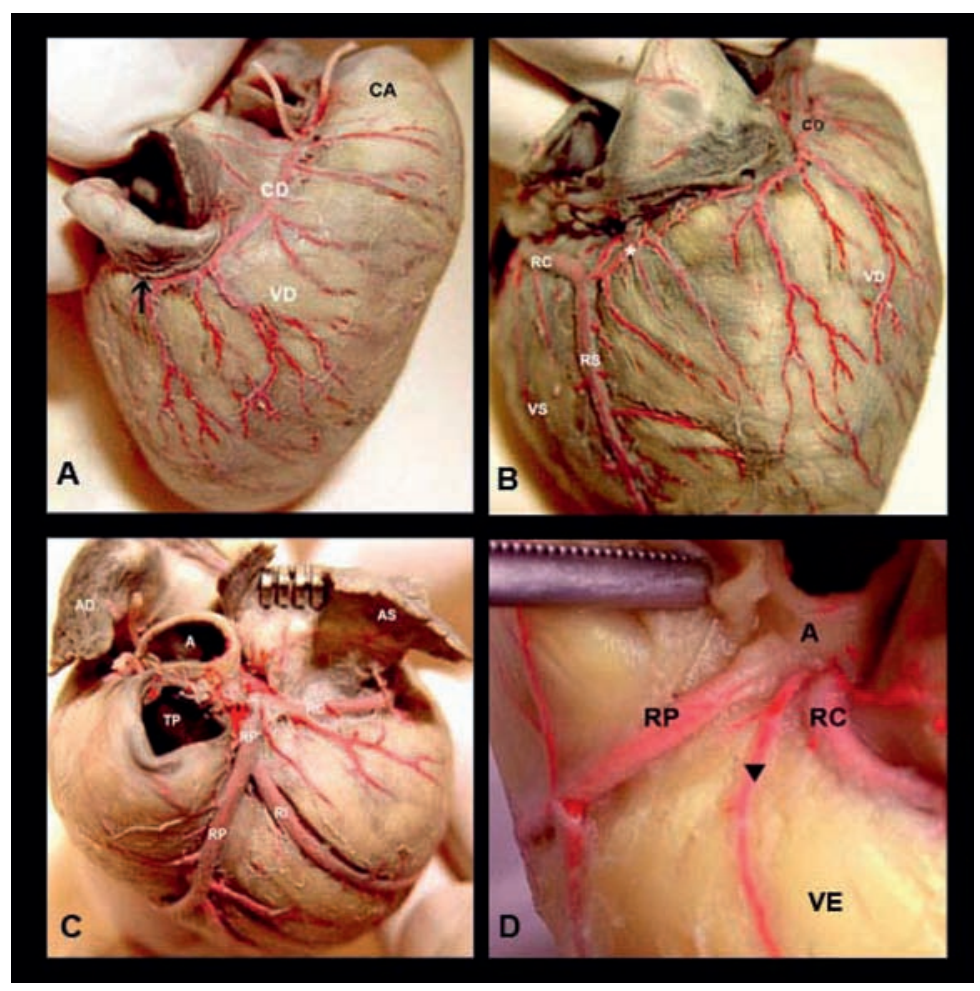

Figuras 1A, 1B, 1C e 1D - Fotografia de corações de capivara (Hydrochaeris hydrochaeris). 1A - face atrial onde se observam cone arterioso (CA), ventrículo direito (VD), artéria coronária direita (CD) e ramo circunflexo (seta) que irá se anastomosar com o ramo subsinuoso (RS) da artéria coronária esquerda. 1B - margem ventricular direita onde se observam o ventrículo direito (VD), ventrículo esquerdo (VS), ramo circunflexo (RC) e ramo subsinuoso (RS) da artéria coronária esquerda, estando este último ramo anastomosando-se $\left(^{*}\right)$ com a artéria coronária direita (CD).1C - face auricular, onde se observam aorta (A), tronco pulmonar (TP), aurícula direita (AD) e aurícula esquerda (AS). A artéria carótida esquerda dividi-se inicialmente em ramo interventricular paraconal $(\mathrm{RP})$, que dá origem ao ramo intermédio (RI) e ramo circunflexo (RC). 1D - face auricular onde se observam ventrículo esquerdo (VE) e aorta (A) dando origem ao ramo interventricular paraconal (RP), ramo intermédio $(\boldsymbol{\nabla})$ e ramo circunflexo $(\mathrm{RC})$ da artéria coronária esquerda

co coronário e emitindo ramos para a margem ventricular direita (Figuras 1A e B). Na face atrial ela apresenta ramo anastomótico, em todos os casos, como descrito anteriormente.

\section{Discussão}

Em 96,6\% dos casos as capivaras apresentaram duas artérias coronárias, esquerda e direita, com origem a partir da aorta ascendente, fato que também foi observado por Borelli, Fernandes Filho e Ferrei$\mathrm{ra}^{1}$, Vicentini, Orsi e Dias ${ }^{3}$, Banchi $^{7}$, Bossi $^{8}$, Montané e Bourdelle ${ }^{9}$, Vischia ${ }^{10}$, Mannu ${ }^{11}$, Martin e Schauder ${ }^{12}$, Barone e Colin ${ }^{13}$, Hegazi ${ }^{14}$, Finelli ${ }^{15}$, Gonzales y Garcia e Gonzalez Alvarez ${ }^{16}$, Bertho ${ }^{17}$, Martini ${ }^{18}$, Koch ${ }^{19}$,
Schwarze e Schröder ${ }^{20}$, Frink e Merrik ${ }^{21}$, Hoffmann ${ }^{22}$, Bruni e Zimmerl ${ }^{23}$, Ellenberger e Baum ${ }^{24}$, Ghoshal ${ }^{25}$, Nickel, Schummer e Seiferle ${ }^{26}$, Godinho, Cardoso e Nascimento $^{27}$, Dyce, Sack e Wensing ${ }^{28}$, Evans ${ }^{29}$, Cos$\operatorname{ta}^{30}$ e Lourenço ${ }^{31}$.

Cervený e $\operatorname{Kaman}^{32}$, estudando bovinos jovens, relatam a ausência da artéria coronária esquerda, observação não evidenciada nos corações das capivaras aqui estudadas. Quanto à observação de não estar presente a artéria coronária esquerda, mas sim os seus ramos, ramo interventricular paraconal e circunflexo, foi também relatado por Vicentini, Orsi e Dias ${ }^{3}$ que evidenciaram em cobaia quatro ramos possuindo origem a partir da aorta. 
Relativo ao diâmetro entre as artérias coronárias observou-se em corações de capivara que a artéria coronária esquerda apresenta um diâmetro maior que a coronária direita.

Uma vez a artéria coronária esquerda estando presente, esta se divide em ramo interventricular paraconal e ramo circunflexo, como também foi relatado por Mannu ${ }^{11}$, Barone e Colin ${ }^{13}$, Hegazi ${ }^{14}$, Finelli ${ }^{15}$, Gonzales y Garcia e Gonzalez Alvarez ${ }^{16}$, Martini $^{18}$, Schwarze e Schröder ${ }^{20}$, Frink e Merrik ${ }^{21}$, Nickel, Schummer e Seiferle ${ }^{26}$ e Lourenço ${ }^{31}$.

Nos corações de capivara estudados neste trabalho, evidenciou-se o ramo intermédio tendo origem a partir do ramo circunflexo da artéria coronária esquerda, fato também relatado por Finelli ${ }^{15}, \mathrm{Koch}^{19}$ e Lourenço ${ }^{31}$. Estes autores citaram o ramo, mas nomearam de maneiras distintas deste trabalho. Preferiu-se seguir a Nomina Anatomica Veterinaria ${ }^{4,5,6}$ que o nomeia como ramo intermédio.

Quanto às anastomoses encontradas entre as artérias coronárias esquerda e direita nos corações de ca-

\section{Referências}

1.BORELLI, V.; FERNANDES FILHO, A.; FERREIRA, N. Sobre a origem do ramus descendens subsinuosus em búfalos sem definição racial. In: CONFERENCIA ANUAL DA SOCIEDADE PAULISTA EM MEDICINA VETERINÁRIA, 14., 1973, São Paulo. Anais... 1973.

2.BEZERRA, A. J. C.; DIDIO, A. S.; DI DIO, L. J. A.; HARDIN, C. J. Coronary arteries and their main branches in Camelus dromedaries. Quaderni di Anatomia Pratica, v. 40, p. 1-4, 1984.

3.VICENTINI, C. A.; ORSI, A. M.; DIAS, S. M. Anatomical observations of the coronary artery vascularization in the guinea pigs (Cavia porcellus, L.). Anatomischen Anzeiger, v. 172, n. 3, p. 209-212, 1991.

4.INTERNATIONAL COMMITTEE ON VETERINARY GROSS ANATOMICAL NOMENCLETURE. Nomina anatomica veterinaria. 4 . ed. Zurich, 1994. (Together with nomina histological, 2. ed. 1992 and nomina embriologica veterinária, 1992).

5.INTERNATIONAL COMMITTEE ON VETERINARY EMBRIOLOGICAL NOMENCLATURE. Nomina embryologica. Zurich, 1994. (Together with nomina anatomica veterinaria, 4. ed. and nomina histologica, 2. ed. rev.).

6.INTERNATIONAL COMMITTEE ON VETERINARY HISTOLOGICAL NOMENCLETURE. Nomina histológica. 2. ed. rev. Zurich, 1994.(Together with nomina anatomica veterinaria, 4. ed. 1992 and nomina embriologica veterinária). pivara, relatos semelhantes foram feitos por Finelli ${ }^{15}$, Gonzales y Garcia e Gonzalez Alvarez ${ }^{16}$, Martini ${ }^{18}$, Koch $^{19}$, Schwarze e Schröder ${ }^{20}$, Ghoshal ${ }^{25}$, Godinho, Cardoso e Nascimento ${ }^{27}$ e Dyce, Sack e Wensing ${ }^{28}$.

\section{Conclusões}

Com o material aqui estudado pode-se concluir que: as artérias coronárias esquerda e direita estão presentes em $96,6 \%$ dos casos e em 3,3\% dos casos os ramos interventricular paraconal e circunflexo da artéria coronária esquerda possuem origem diretamente da aorta ascendente; em 100\% dos casos a artéria coronária esquerda possui diâmetro e área de irrigação maior que a artéria coronária direita; ocorre anastomose entre o ramo circunflexo da artéria coronária esquerda e a artéria coronária direita.

\section{Agradecimentos}

Os autores agradecem a FAPESP (Proc. 02/07409-7) pelo auxílio financeiro.

7.BANCHI, A. Morfologia delle arteriae coronaries cordis. Archivio Italiano di Anatomia e di Embriologia, v. 3, p. 154162,1904

8.BOSSI, V. Angelology In: BOSSI, V.; CARADONNA, G. B.; SPAMPANI, G.; VARALDI, L.; ZIMMERL, U. Tratado de anatomia veterinária. Milano: Francesco Valardes, 1909. v. 2, p. 77-79.

9.MONTANÉ, L.; BOURDELLE, E. Anatomia régionale des animaux domestiques. II Ruminants. Paris: J.B. Librairie, 1917. v. 2, p. 243-247.

10.VISCHIA, R. Beitrag zum studim der arterial coronariae cordis. Anatomischen Anzeiger, v. 16, p. 487-491, 1926.

11. MANNU, A. Apparecchio vascolare. In: ZIMMERL, V. Trattadi anatomia veterinaria. Milano: Francesco Vallardi, 1930. p. 30 .

12.MARTINS, P.; SCHAUDER, W. Lehrbuch der Anatomie der Haustiere. Stuttgart: Verlag von Schickhardt \& Ebner, 1938. v. 3, pt. 3, p. 388.

13. BARONE, R.; COLIN A. Les artère du coeur chez les ruminants domestiques. Révue du Medicine Véterinaire, v. 102, p. 172$181,1951$.

14.HEGAZI, H. Die blutgefässversorgung des herzens von rind, schaf und ziege. 1958. 958 p. Thesis (Doktorgrades) Sachbereish Veterinärmedizin und Tiervuzht der Justus Liebig Universität, Giessen, 11958. 
15. FINELLI, R. Prime Osservazione sul circolo arterioso coronarico in alcuni ruminanti. Bolletin della Societá di Biologia Sperimentale, v. 1, n. 882, p. 335-336, 1960.

16.GONZALEZ Y GARCIA, J.; GONZALEZ ALVAREZ, R. Anatomia comparada de los animales domesticos. 7. ed. Madrid: Canales, 1961. p. 594.

17. BERTHO, E. Anatomie comparée normale des artères et des veines coronaires du coeur de differents espèces animaux. Archives d'Anatomie, d'Histologie et d'Embriologie, v. 47, p. 283, 1964.

18. MARTINI, E. La vascolarizzazione arteriosa del cuore di alcuni mammiferi domestici. Archivio Italiano di Anatomia e di Embriologia, v. 70, n. 10, p. 352-362, 1965.

19. KOCH, T. Lehrbuch der Veterinär Anatomie. Jena: Gustav Fischer Verlag, 1965. p. 43.

20.SCHWARZE, E.; SCHRÖDER, L. Compendio de anatomia veterinária. Zaragoza: Acribia, 1972.

21.FRINK, R. J.; MERRIK, B. The sheep heart: coronary and conduction system anatomy with special reference to the presence of an os cordis. Anatomical Record, v. 179, n. 8, p. 189-200, 1974

22. HOFFMANN, V. Die Blutgefässversorgung des Pferdeherfens, zugleich auch eine Vergleichende Betrachtung der Topographie der Herzeigenen Blutgesfässe der Haussäuger (Fleischfresser, Schwein und Wiederkaäver). Anatomischen Anzeiger, v. 137, n. 7, p. 79-109, 1975.

23.BRUNI, A. C.; ZIMMERL, U. Anatomia debele animai domestica. Milano: Francesco Valardes, 1977. v. 2, p. 314-316.

24.ELLEMBERGER, W.; BAUM, H. Handbuch de verglichenden anatomie der haustiere. Berlin: Springer Verlag, 1977. p. 629.
25.GHOSHAL, N. G. Coração e artéria das ruminantes. In GETTY, R. SISSON/GROSSMAN Anatomia dos animais domésticos. 5. ed. Rio de Janeiro: Interamericana, 1981. v. 1, p. $1847-1848$.

26. NICKEL, R.; SCHUMMER, A.; SEIFERLE, E. The anatomy of the domestic animals. In: SCHUMMER, A.; WILKENS, H.; VOLLMERHAUS, B.; HABERMEHL, K-H. The anatomy of the domestic animals. The circulatory system, the skin and the cutaneous organs of the domestic mammals. Berlin; Hamburg: Paul Perey, 1981. v. 3, p. 38-40.

27.GODINHO, H. P.; CARDOSO, F. M.; NASCIMENTO. J. F. Anatomia dos ruminantes domésticos. Belo Horizonte: Universidade Federal de Minas Gerais, 1987. p. 338-339.

28.DYCE, K. M.; SACK, W. O.; WENSING, C. J. G. Tratado de anatomia veterinária. Rio de Janeiro: Guanabara Koogan, 1990. p. 151-152.

29.EVANS, H. E. Miller's anatomy of the dog. 3. ed. London: W.B.Saunders Company, 1993. p. 598-600.

30. COSTA, J. B. Contribuição ao estudo da vascularização arterial do coração de caprinos da raça Bojo Brasileira. São Paulo, 1995. 74 p. Dissertação (Mestrado) - Faculdade de Medicina Veterinária e Zootecnia, Universidade de São Paulo, São Paulo, 1995.

31.LOURENÇO, M. G. Vascularização arterial dos músculos papilares do ventrículo esquerdo de cães (Canis familiaris, L. 1758). 2003. 96 p. Dissertação (Mestrado) - Faculdade de Medicina Veterinária e Zootecnia, Universidade de São Paulo, São Paulo, 2003.

32.CERVEÝ, C.; KAMAN, J. Common stem of coronary arteries in a calf. Anatomischen Anzeiger, v. 113, n. 6, p. 29-35, 1963. 